\title{
Single-Stage Endoscopic Stone Extraction and Cholecystectomy during the Same Hospitalization: What Is the Optimal Strategy for Patients with Choledocholithiasis and Cholelithiasis?
}

\author{
Tae Yoon Lee \\ Division of Gastroenterology, Department of Internal Medicine, Konkuk University School of Medicine, Seoul, Korea
}

See "Single-Stage Endoscopic Stone Extraction and Cholecystectomy during the Same Hospitalization" by Toshiaki Terauchi, Hiroharu Shinozaki, Satoshi Shinozaki, et al., on page 59-64.

Up to $18 \%$ of patients who undergo cholecystectomy for gallstones have concomitant choledocholithiasis; thus, these patients are referred for endoscopic retrograde cholangiopancreatography (ERCP) and cholecystectomy. However, practice patterns in patients with choledocholithiasis and cholelithiasis are still largely institution-based, depending on the institutional resource constraints. Some have advocated combining ERCP with cholecystectomy during the same admission, while others have argued that ERCP and cholecystectomy should be delayed to minimize postoperative adverse events. ${ }^{1}$ Although single-stage endoscopic stone extraction is accepted as the standard strategy for treatment of patients with choledocholithiasis, some endoscopists, especially in Japan, still prefer to perform endoscopic drainage and stone removal separately. ${ }^{2}$

In this issue of Clinical Endoscopy, Terauchi et al. compared the clinical outcomes of subjects who underwent cholecystectomy at index admission $(n=106)$ or during a subsequent hospitalization $(n=13){ }^{2}$ They also investigated the efficacy and

Received: December 10, 2018 Accepted: December 10, 2018

Correspondence: Tae Yoon Lee

Division of Gastroenterology, Department of Internal Medicine, Konkuk University School of Medicine, 120-1 Neungdong-ro, Gwangjin-gu, Seoul 05030, Korea Tel: +82-2-2030-7497, Fax: +82-2-2030-7458, E-mail: widebrow@empal.com ORCID: https://orcid.org/0000-0003-1008-9814

(c) This is an Open Access article distributed under the terms of the Creative Commons Attribution Non-Commercial License (http://creativecommons.org/ licenses/by-nc/3.0) which permits unrestricted non-commercial use, distribution, and reproduction in any medium, provided the original work is properly cited. safety of single-stage ERCP. Although there is no consensus regarding the optimal timing for cholecystectomy after endoscopic stone removal, the most time-efficient approach is performing ERCP and cholecystectomy during the same hospitalization.

A recent study from the US compared the clinical outcomes of 4,516 patients who underwent cholecystectomy at index admission (41.2\%), elective cholecystectomy within 60 days after discharge (10.9\%), or no cholecystectomy (48.0\%) and evaluated the incidence of recurrent biliary events, mortality, and cost. ${ }^{3}$ Surprisingly, nearly half of the patients did not undergo a subsequent cholecystectomy during or after hospitalization. Early cholecystectomy was protective against the relative risk of recurrent biliary events within 2 months by $92 \%$, compared with delayed or no cholecystectomy $(p<0.001)$. Two months after the initial discharge, the delayed cholecystectomy group had an $88 \%$ lower risk of recurrent biliary events than the no cholecystectomy group $(p<0.001)$. Although either early or delayed cholecystectomy reduces recurrent biliary events, the delayed cholecystectomy group showed a 10-fold higher risk of recurrent biliary events while waiting for an interval cholecystectomy than that in the early cholecystectomy group. Unlike previous studies, Terauchi et al. showed that there were no significant differences between the early and delayed cholecystectomy groups in terms of operative time, rate of postoperative complications, and interval from cholecystectomy to discharge and could not demonstrate that early cholecystectomy is superior to delayed cholecystecto- 
my. ${ }^{2}$ This result is mostly due to the small number of patients in the delayed cholecystectomy group to draw a meaningful conclusion. Large-scale studies are necessary to confirm these preliminary results.

This study also showed that single-stage stone extraction by ERCP in patients with mild to moderate cholangitis due to choledocholithiasis is safe and feasible, indicating a satisfactory rate of complete stone removal (92\%) and an acceptable complication rate (3\%). Undoubtedly, single-stage endoscopic stone removal is generally recommended for choledocholithiasis with the exception of cases of severe cholangitis. In patients with grade III cholangitis based on the 2018 Tokyo guideline, endoscopic or percutaneous biliary drainage without stone removal is better to complete the procedure as soon as possible. ${ }^{4}$ Saito et al. reported that the complication rates for ERCP were similar between the patients who underwent single-stage endoscopic stone removal and those who underwent two-stage stone removal $(13.2 \%$ vs. $7.4 \%, p=0.11)$ in patients with choledocholithiasis. ${ }^{5}$ In the single-stage group, the proportion of patients with a hospital stay of within 7 days was significantly higher $(56.5 \%$ vs. $13.9 \%, p<0.001)$ and the number of ERCP attempts was significantly lower than that in the two-stage group. Thus, single-stage endoscopic stone removal in patients with non-severe cholangitis should be a standard treatment because it can reduce the hospital stay and medical costs.
To summarize, the benefit of single-stage endoscopic stone removal and cholecystectomy during the same hospitalization is obvious, and multiple additional sessions of ERCP and a long interval between ERCP and cholecystectomy increase medical costs or the likelihood of recurrent biliary events. Readers should remember the findings of the current study and proceed with both procedures simultaneously or in rapid sequence unless medical comorbidities preclude it.

Conflicts of Interest

The author has no financial conflicts of interest.

\section{REFERENCES}

1. Reinders JS, Kortram K, Vlaminckx B, van Ramshorst B, Gouma DJ, Boerma D. Incidence of bactobilia increases over time after endoscopic sphincterotomy. Dig Surg 2011;28:288-292.

2. Terauchi TT, Shinozaki H, Shinozaki S, et al. Single-stage endoscopic stone extraction and cholecystectomy during the same hospitalization. Clin Endosc 2019;52:59-64.

3. Huang RJ, Barakat MT, Girotra M, Banerjee S. Practice patterns for cholecystectomy after endoscopic retrograde cholangiopancreatography for patients with choledocholithiasis. Gastroenterology 2017;153:762-771.e2.

4. Miura F, Okamoto K, Takada T, et al. Tokyo guidelines 2018: initial management of acute biliary infection and flowchart for acute cholangitis. J Hepatobiliary Pancreat Sci 2018;25:31-40.

5. Saito H, Kadono Y, Kamikawa K, et al. The incidence of complications in single-stage endoscopic stone removal for patients with common bile duct stones: a propensity score analysis. Intern Med 2018;57:469-477. 